\title{
Clinicopathological characteristics and recurrence risk of papillary thyroid microcarcinoma in the elderly
}

This article was published in the following Dove Press journal: Cancer Management and Research

\author{
Xiaofei Wang* \\ Jianyong Lei* \\ Tao Wei \\ Jingqiang Zhu \\ Zhihui Li
}

Department of Thyroid \& Parathyroid Surgery, West China Hospital, Sichuan University, Chengdu, People's Republic of China

*These authors contributed equally to this work.
Correspondence: Zhihui Li Department of Thyroid \& Parathyroid Surgery, West China Hospital, Sichuan University, No. 37 GuoXueXiang, Chengdu, People's Republic of China Tel +861 8980602027

Email westchinalzh@।63.com
Background: The optimal management for elderly patients with papillary thyroid microcarcinoma (PTMC) is not well established. The aim of the present study is to describe the clinicopathological characteristics and identify predictors of recurrence in the elderly PTMC patients.

Methods: We conducted a retrospective study of PTMC patients who underwent thyroidectomy. The clinicopathological characteristics were compared between patients with age $\geq 65$ years and $<65$ years. The independent predictors of recurrence were identified by multivariate Cox regression analysis.

Results: The patients in elderly group were more likely to exhibit bilaterality $(P<0.050)$, extrathyroidal extension $(P<0.001)$, and central compartment lymph node metastasis (CLNM) $(P<0.010)$, especially the ratio of CLNM $>0.5(P<0.010)$, than the patients with age $<65$ years. The elderly patients had poorer recurrence-free survival (RFS) than those with younger ages $(P=0.002)$. Multivariate analysis indicated tumor size $>5 \mathrm{~mm}(P=0.009)$ and CLNM ratio $>0.5(P=0.002)$ were independent risk factors for recurrence of the elderly patients with PTMC.

Conclusions: The elderly PTMC patients have more aggressive biological characteristics and higher recurrence rate. More aggressive treatment and rigorous follow-up could be considered for elderly patients with tumor diameter $>5 \mathrm{~mm}$ and CLNM ratio $>0.5$.

Keywords: papillary thyroid microcarcinoma, recurrence, elderly

\section{Introduction}

Papillary thyroid microcarcinoma (PTMC) is defined as a PTC with no more than 1 $\mathrm{cm}$ at the greatest dimension, and its incidence has increased dramatically in recent years. ${ }^{1}$ PTMC accounts for up to $40 \%$ of new thyroid cancer diagnoses worldwide and has an excellent prognosis. ${ }^{2}$ However, approximately $30-70 \%$ and $1-3 \%$ of patients with PTMC who have cervical lymph node metastasis and distant metastases at diagnosis have worse prognosis, with high rates of recurrence. ${ }^{3}$ Therefore, the optimal treatment strategy of PTMC remains controversial. ${ }^{4}$

Given the favorable prognosis, several kinds of research from Japan proposed that an active surveillance was a safe and effective alternative to immediate surgery for properly selected patients with PTMC. ${ }^{5-7}$ Moreover, one study indicated that elderly PTMC patients may be the best candidates for observation because of its lower risk of progress. ${ }^{8}$ In addition, latent PTMC was detected at autopsy in $0.5-5.2 \%$, and the incidence had a peak in the elderly. ${ }^{9,10}$ These evidence suggest 
that relatively conservative treatment procedures might be reasonable for the elderly PTMC.

On the other hand, a large number of studies have shown that age is an important prognostic factor for PTC and has been used as a major stratification factor in prominent classification systems such as TNM, AMES (Age, Metastases, Extent, Size), and MACIS (Metastases, Age, Completeness of resection, Invaision, Size). ${ }^{11-13}$ Old age is associated with higher recurrence and death risk in patients with PTC. Therefore, a more aggressive treatment for elderly patients has been advocated by other authors. ${ }^{14,15}$ It has been reported that PTMC may have a similar recurrence rate to that of their clinically detectable counterparts. ${ }^{16,17}$ And of course, all cases of advanced PTC progressed from PTMC. Thus, a more aggressive treatment seems to be suitable to elderly patients with PTMC. However, uncertainty still exists due to the absence of evidence on the recurrence of PTMC in the elderly. Recently, Tang et $\mathrm{al}^{18}$ showed that $>65$ years elderly PTMC patients had a lower cause-specific survival, but the study was based on the data from the SEER database and could not analyze the recurrence risk. PTMC causes very few disease-specific deaths, but the recurrence risk has been reported as high as $40 \%$ in the literature. ${ }^{19}$ Treatment for postoperative recurrence may affect patients' quality of life and increase public health care burden. Therefore, the detection of postoperative recurrence may be valuable than disease-specific mortality.

In the present study, we retrospectively collected the data of PTMC patients who underwent thyroidectomy in our hospital and analyzed the influence of old age on the risk of postoperative recurrence, providing evidence-based evidence for the optimal treatment policy of elderly PTMC.

\section{Patients and methods}

\section{Patients}

We retrospectively reviewed the medical records of all consecutive PTMC patients who underwent initial thyroid surgery at our center from January 2012 to January 2015. Patients aged $<18$ years $(n=2)$, lateral cervical lymph node metastasis at the time of initial diagnosis $(n=54)$, or follow-up duration of $<6$ months $(n=32)$ were excluded. Recurrence $(n=10)$ diagnosed within 3 months after surgery was also excluded due to the possibility of persistence disease. Ultimately, 1,235 patients were enrolled in this study and were evaluated to identify the risk factors of postoperative recurrence. According to whether the patient was $>65$ years old at the time of diagnosis, 1,235 patients were divided into two groups: elderly (202 patients, $16.4 \%$ ) and nonelderly groups (1,033 patients, $83.6 \%)$.

\section{Treatment}

The management of patients with PTC has been described previously. ${ }^{20}$ Briefly, neck ultrasonography (US) was routinely performed in all patients preoperatively. When a highly suspicious thyroid nodule was found, the nodule was further evaluated by US-guided fine-needle aspiration (FNA) cytology examination. Patients with cytological diagnosis of PTMC received surgical therapy or active surveillance depending on patient preferences and the decision of the surgeon. As for the surgical extent, lobectomy (LT) was only performed for confirmed PTMC without bilateral foci, extrathyroidal extension (ETE), lymph node metastasis, and distant metastasis; otherwise, total thyroidectomy (TT) was performed. A complete ipsilateral central compartment lymph node dissection (CND) was performed for patients who underwent LT. Ipsilateral or bilateral CND was performed for patients who underwent TT. After operation, all patients were treated with levothyroxine for TSH suppression therapy. Some patients who underwent TT received radioactive iodine (RAI) ablation therapy according to the recommendation of American Thyroid Association guidelines. $^{21}$

\section{Follow-up}

Patients were followed up every 3 months during the first year and at 6-month intervals thereafter. Patients received regular physical examinations, neck US, serum free thyroxine (FT4), thyrotropin (TSH), thyroglobulin (Tg), and antithyroglobulin antibody ( $\mathrm{TgAb}$ ) measurements. Any suspicious lesions were assessed using US-guided FNA cytology and imaging modalities, including US, contrast-enhanced computed tomography, whole-body nuclear scanning with radioactive iodine, and/or 18F-fluorodeoxyglucose positron emission tomography/computed tomography. Recurrence was defined as structural recurrence, determined using imaging modalities followed by histological confirmation, regardless of serum $\mathrm{Tg}$ levels. Recurrence-free survival (RFS) was defined as the time interval between initial surgery and the detection of structural recurrent disease or the last neck US and cross-sectional imaging. The overall median follow-up time was 38 months (IQR, 16 to 44 months).

\section{Statistical analyses}

Continuous variables were presented as mean $\pm \mathrm{SD}$, and categorical variables were presented as number with percentage. The clinicopathological characteristic difference between 
elderly and nonelderly was assessed using Mann-Whitney $U$ test, Chi-square, or Fisher exact test according to the distribution of the variables. Multivariate Cox proportional hazard models were used to identify independent predictors of postoperative recurrence through the backward elimination of variables with $P$-values lower than 0.1 in univariate analyses. The estimated HR and 95\% CI were calculated. RFS curves were constructed using the Kaplan-Meier method, and differences were evaluated by log-rank test. $P$-values $<0.05$ were considered statistically significant. All $P$-values were two-sided. All analyses were performed using SPSS ver. 22.0 (IBM Corp., Armonk, NY, USA).

\section{Ethics approval}

The Institutional Review Board of West China Hospital of Sichuan University waived approval for this study due to the retrospective and fully anonymized character. All patient data were kept strictly confidential, and all procedures performed in this study were in accordance with the Declaration of Helsinki.

\section{Results}

Clinicopathological characteristics were compared between patients aged $\geq 65$ years (elderly group) and aged $<65$ years (nonelderly group) at the time of diagnosis, as presented in Table 1. Compared with nonelderly patients, patients in the elderly group were more likely to exhibit bilaterality (17.8\% vs $11.2 \%, P=0.011$ ), ETE ( $49.5 \%$ vs $32.2 \%, P<0.001)$, and central compartment lymph node metastasis (CLNM) (55.4\% vs $43.3 \%, P=0.002$ ), especially the CLNM ratio (defined as the number of metastatic lymph nodes in the central compartment on pathology divided by the total number of harvested central compartment lymph nodes) $>0.5$ (16.3\% vs $7.7 \%, P=0.006)$.

During the follow-up period, 40 patients $(3.2 \%)$ had recurrences, $12(5.9 \%)$ in the elderly group and 28 $(2.7 \%)$ in the nonelderly group. Among them, cervical lymph node recurrence (CLNR) was diagnosed in 38 $(3.06 \%)$ patients and lung metastasis (LM) in one $(0.08 \%)$ patient; one $(0.08 \%)$ patient had CLNR and LM. No recurrence was observed in the thyroidal bed (Table 1). In the unadjusted analysis, 5-year RFS estimates were inversely associated with increased patient age: $95 \%$ for patients with age $<65$ years and $92 \%$ for patients with age $\geq 65$ years (Figure 1). The elderly patients had poorer RFS than younger patients $(P=0.002)$. Because no death was observed in our
Table I Clinicopathological characteristics of patients with PTMC

\begin{tabular}{|c|c|c|c|}
\hline Variables & $\begin{array}{l}\text { Elderly } \\
(n=202)\end{array}$ & $\begin{array}{l}\text { Nonelderly } \\
(n=1033)\end{array}$ & $P$-value \\
\hline $\begin{array}{l}\text { Mean age at diagnosis, } \\
\text { years }\end{array}$ & $67.8 \pm 4.02$ & $42.2 \pm 9.76$ & $0.000 *$ \\
\hline Female sex, n (\%) & $152(75.2)$ & $765(74.1)$ & 0.926 \\
\hline $\begin{array}{l}\text { Hashimoto's thyroiditis, } \\
\text { n (\%) }\end{array}$ & $35(17.3)$ & $201(19.5)$ & 0.438 \\
\hline Nodular goiters, n (\%) & $52(25.7)$ & $243(23.5)$ & 0.557 \\
\hline Mean tumor size $(\mathrm{mm})$ & $7.6 \pm 1.92$ & $7.3 \pm 1.82$ & 0.802 \\
\hline $\begin{array}{l}\text { Tumor size >5mm, } \\
\mathrm{n}(\%)\end{array}$ & $168(83.2 \%)$ & $845(81.8)$ & 0.894 \\
\hline Bilaterality, n (\%) & $36(17.8)$ & $116(11.2)$ & $0.011 *$ \\
\hline Multifocality, n (\%) & $72(35.6)$ & $322(3 \mid .2)$ & 0.255 \\
\hline $\begin{array}{l}\text { Extrathyroidal exten- } \\
\text { sion, } n(\%)\end{array}$ & $100(49.5)$ & $333(32.2)$ & $0.000 *$ \\
\hline $\begin{array}{l}\text { BRAF mutation/total } \\
\text { n (\%) }\end{array}$ & $\begin{array}{l}48 / 68 \\
(70.6)\end{array}$ & $207 / 314(65.9)$ & 0.459 \\
\hline TT, n (\%) & $176(86.1)$ & $836(80.9)$ & 0.078 \\
\hline BCND, n (\%) & $76(37.6)$ & $378(36.6)$ & 0.873 \\
\hline T4a, n (\%) & $3(1.5)$ & $12(1.2)$ & 0.701 \\
\hline CLNM, n (\%) & III (55.4) & $447(43.3)$ & $0.002 *$ \\
\hline $\begin{array}{l}\text { CLNM number }>5 \text {, } \\
\text { n (\%) }\end{array}$ & $27(13.4)$ & $102(9.9)$ & 0.716 \\
\hline CLNM ratio >0.5, n (\%) & $33(16.3)$ & $80(7.7)$ & $0.006 *$ \\
\hline RAI, n (\%) & $80(39.6)$ & $48 \mid(46.6)$ & 0.051 \\
\hline Recurrence, n (\%) & $12(5.9)$ & $28(2.7)$ & $0.018 *$ \\
\hline CLNR & II (4.5) & $27(2.6)$ & $0.033^{*}$ \\
\hline LM & $0(0.5)$ & I $(0.1)$ & 1.000 \\
\hline CLNR + LM & $\mathrm{I}(\mathrm{I} .0)$ & $0(0.0)$ & 0.363 \\
\hline $\begin{array}{l}\text { Median follow-up time, } \\
\text { months (IQR) }\end{array}$ & $37(2 \mid-42)$ & $38(22-45)$ & 0.778 \\
\hline
\end{tabular}

Notes: $* P<0.05$; bold values indicate statistical signficance.

Abbreviations: PTMC, papillary thyroid microcarcinoma; TT, total thyroidectomy; BCND, bilateral central compartment lymph node dissection; CLNM, central compartment lymph node metastasis; RAI, radioactive iodine; CLNR, cervical lymph node recurrence; LM, lung metastasis.

cohort, disease-specific survival could not be analyzed between groups.

In multivariate analysis by Cox proportional hazard regression models, the independent predictors of recurrence were age (HR 1.03, 95\% CI: $1.01-1.07 ; P=0.004)$, CLNM ratio $>0.5$ (HR 3.38, 95\% CI: 1.58-7.24; $P=0.002$ ), ETE (HR 2.24, 95\% CI: $1.30-4.63 ; P=0.003$ ), and tumor size $>5 \mathrm{~mm}$ (HR 3.59, 95\%CI: 2.19-5.87; $P<0.001$ ) for all the PTMC patients; CLNM ratio $>0.5$ (HR 1.91, 95\% CI: 1.17-3.11; $P=0.009$ ) and ETE (HR 2.37, 95\% CI: $1.17-5.79 ; P=0.010$ ) for the younger patients; CLNM ratio $>0.5$ (HR 3.52, 95\% CI: $1.60-7.75 ; P=0.002)$ and tumor size $>5 \mathrm{~mm}$ (HR 5.62, 95\% CI: $1.76-9.37 ; P=0.009)$ for the elderly patients (Table 2). 


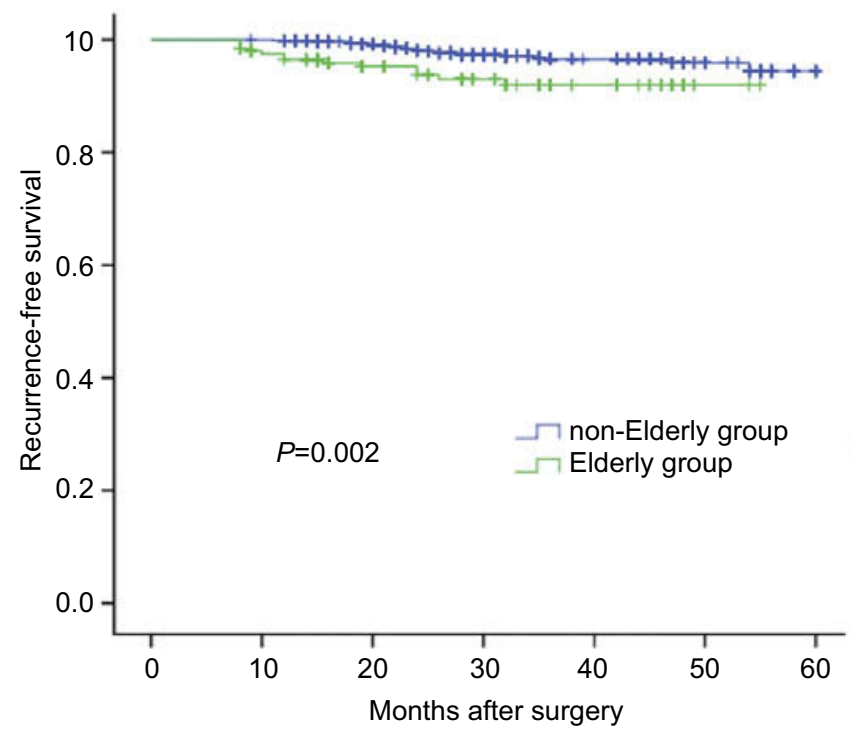

Figure I The elderly PTMC patients had poorer recurrence-free survival than those with younger ages $(P=0.002$ by the log-rank test).

Abbreviation: PTMC, papillary thyroid microcarcinoma.

\section{Discussion}

The rapid aging of the population in many countries and regions around the world has become an important social issue. Recent surveys have indicated that the incidence of PTC is rising more rapidly over age 65 years than those under 65 (annual percentage change, 8.8\% vs 6.4\%) in USA. $^{22}$ In addition, elderly patients may not be good candidates for surgical treatment due to the age-related physiological changes, limited social support, and general status. Therefore, the optimal treatment options for elderly PTMC need to weigh the risks and benefits associated with each treatment.

It is controversial about the impact of age on the PTC prognosis: patient age older than 45 or 55 years has been considered to be a poor prognostic factor by various reports, consistent with risk stratification promoted by American Joint Committee on Cancer, ${ }^{23}$ whereas other authors suggested alternative age cutoffs ranging from 40 to 70 years. Although there is no consensus on a clear definition of "old age", conventionally the elderly has been defined as a chronological age of 65 years or more. ${ }^{24-26}$ As other studies, ${ }^{27-30}$ we chose to use the age limit of 65 years with the aim of obtaining a larger series. These reports described the clinical features or prognosis of elderly patients with PTC, but few reports describe elderly PTMC patients.

In this study, we evaluated the clinicopathological characteristics in a cohort of 1,235 patients with PTMC and found that the elderly PTMC patients had more aggressive tumor biological features, such as bilateral cancer, ETE, and CLNM. Therefore, it is not surprising that the recurrence rate of elderly patients is much higher than that of young patients. In our cohort, the overall relapse rate was $3.2 \%$, which was consistent with that of previously published series reported recurrence rates of between $0.5 \%$ and $7.9 \%{ }^{4}$

Furthermore, we identified independent predictors of recurrence risk in all patients and patients, stratifying based on whether the patient was $>65$ years of age. Our results indicated that there were different predictors of recurrence between young and elderly patients. Recurrence was significantly associated with age, CLNM ratio $>0.5$, ETE, and tumor size $>5 \mathrm{~mm}$ in all PTMC patients. Recurrence was associated with CLNM ratio $>0.5$ and ETE in the younger patients, while it was related with CLNM ratio $>0.5$ and tumor size $>5 \mathrm{~mm}$ in the elderly patients (Table 2).

The rate of CLNM was reported varying from $10 \%$ to $50 \%,{ }^{31}$ in which prophylactic CND had the highest rates. In our cohort, $45 \%$ of PTMC had CLNM, close to the highest reported, probably due to our policy to performing CND for all PTMC according to the Chinese expert consensus and guideline for the treatment of PTMC. ${ }^{32}$ Although there is still controversy regarding the effectiveness of prophylactic CND on decreasing postoperative

Table 2 Multivariate analyses for recurrence of the PTMC patients

\begin{tabular}{|l|l|l|l|l|l|l|}
\hline \multirow{2}{*}{ Variables } & \multicolumn{2}{l|}{ All patients } & \multicolumn{2}{l|}{ Nonelderly group } & \multicolumn{2}{l|}{ Elderly group } \\
\cline { 2 - 7 } & aHR (95\% CI) & P-value & aHR (95\% CI) & P-value & aHR (95\% CI) & $P$-value \\
\hline Age (continuous) & $1.03(1.01-1.07)$ & 0.004 & - & & - & \\
CLNM ratio >0.5 & $3.38(1.58-7.24)$ & 0.002 & $1.91(1.17-3.11)$ & 0.009 & $3.52(1.60-7.75)$ & 0.002 \\
Extrathyroidal extension & $2.24(1.30-4.63)$ & 0.003 & $2.37(1.17-5.79)$ & 0.010 & - & \\
Tumor size >5 mm & $3.59(2.19-5.87)$ & $<0.001$ & - & & $5.62(1.76-9.37)$ & 0.009 \\
\hline
\end{tabular}

Abbreviations: PTMC, papillary thyroid microcarcinoma; aHR, adjusted hazard ratio; HD, CLNM, central compartment lymph node metastasis; RAI, radioactive iodine; ETE, extrathyroidal extension. 
recurrence in patients with $\mathrm{PTMC}$, a recent study found that prophylactic CND does reduce the risk of recurrence in the central compartment and not increase long-term postoperative complications. ${ }^{33}$ It is currently known that the impact of CLNM on recurrence depends on the number, proportion, and size of the metastases, but microscopic nodal positivity does not carry the recurrence risk, ${ }^{34,35}$ which may explain why CLNM is not a significant predictor for recurrence in multivariate analysis. Unfortunately, we were unable to distinguish between micrometastatic and significantly metastatic lymph nodes, because the data on lymph node size were unavailable. In addition, our results indicate that the ratio of CLNM is more valuable than the number of CLNM for predicting recurrence in all the PTMC patients, regardless of patient age.

Previous published studies indicated PTMC with larger tumors was more aggressive (multifocality, ETE, and CLNM) than those with smaller tumors. ${ }^{36,37}$ Our results also indicate that the recurrence risk was significantly higher in elderly PTMC patients with tumor diameter $>5 \mathrm{~mm}$ than those with the diameter $\leq 5 \mathrm{~mm}$. Thus, an immediate surgery should be recommended to elderly patients with tumor diameter $>5 \mathrm{~mm}$, if the patients could tolerate the operation.

ETE included minor ETE and gross ETE. Recent studies have suggested that minor ETE correlates with CLNM but does not correlate with recurrence in multivariate analysis. $^{38,39}$ In our cohort, we failed to differentiate the minor ETE from gross ETE. This may be why the proportion of ETE in our cohort is high (49\% in elderly and 32.3\% in nonelderly patients) and our study found no association between ETE and recurrence in elderly patients. RAI ablation could decrease recurrence and increase overall survival for American Thyroid Association (ATA) high-risk patients with PTC. However, our results noted that RAI almost had no positive treatment effect for PTMC patients. This result is consistent with recent published studies, which reported that RAI had no impact on recurrence in patients with PTMC. ${ }^{40,41}$

A number of studies have suggested that TT can decrease recurrence rates and improve survival rates for PTC, but there were similar clinical outcomes between LT and TT for PTMC. ${ }^{42}$ A study using the SEER database demonstrated TT did not increase cause-specific survival for the elderly PTMC patients. ${ }^{18}$ Consistent with these findings, we found that the extent of surgery was not an independent prognostic factor for elderly patients with PTMC.
This study has several limitations. We cannot extensively distinguish the ETE and CLNM (minor/gross ETE and micro/macrolymph metastases), which would diminish the power of the study. Moreover, the time of follow-up (median: 38 months) is short.However, although PTC may recur a long time after initial treatment, nearly 50\% and more than $75 \%$ of the recurrences were detected within the first 3 and 5 years postoperatively. ${ }^{43}$ In addition, although the number of elderly PTMC patients was large, the number of recurrences was limited due to the lower recurrence rate. Thus, further studies with larger series of patients are needed.

In conclusion, compared to patients with younger age, the elderly PTMC patients have more aggressive biological characteristics, higher recurrence rate, and poorer RFS. Tumor diameter $>5 \mathrm{~mm}$ and CLNM ratio $>0.5$ were independent risk factors for recurrence of elderly patients with PTMC. More aggressive treatment or more rigorous follow-up could be considered for patients with those unfavorable features.

\section{Acknowledgments}

This study was supported by Key Project of Sichuan Provincial Education Department (17ZA0170), Project of Sichuan Health and Family Planning Commission (17PJ105), and Project of Sichuan Provincial Cadre Health Department (2018-107).

\section{Author contributions}

All authors contributed to data analysis, drafting or revising the article, gave final approval of the version to be published, and agree to be accountable for all aspects of the work.

\section{Disclosure}

The authors report no conflicts of interest in this work.

\section{References}

1. Chen AY, Jemal A, Ward EM. Increasing incidence of differentiated thyroid cancer in the United States, 1988-2005. Cancer. 2009;115 (16):3801-3807. doi:10.1002/cncr.24416

2. Davies L, Welch HG. Current thyroid cancer trends in the United States. JAMA Otolaryngol Head Neck Surg. 2014;140(4):317-322. doi:10.1001/jamaoto.2014.1

3. Bernet V. Approach to the patient with incidental papillary microcarcinoma. J Clin Endocrinol Metab. 2010;95(8):3586-3592. doi:10.1210/jc.2010-0698

4. Leboulleux S, Tuttle RM, Pacini F, Schlumberger M. Papillary thyroid microcarcinoma: time to shift from surgery to active surveillance? Lancet Diabetes Endocrinol. 2016;4(11):933-942. doi:10.1016/ S2213-8587(16)30180-2 
5. Ito Y, Uruno T, Nakano K, et al. An observation trial without surgical treatment in patients with papillary microcarcinoma of the thyroid. Thyroid. 2003;13(4):381-387. doi:10.1089/ 105072503321669875

6. Oda H, Miyauchi A, Ito Y, et al. Incidences of unfavorable events in the management of low-risk papillary microcarcinoma of the thyroid by active surveillance versus immediate surgery. Thyroid. 2016;26 (1):150-155. doi:10.1089/thy.2015.0313

7. Sugitani I, Toda K, Yamada K, Yamamoto N, Ikenaga M, Fujimoto Y. Three distinctly different kinds of papillary thyroid microcarcinoma should be recognized: our treatment strategies and outcomes. World J Surg. 2010;34(6):1222-1231. doi:10.1007/s00268-009-0359-x

8. Ito Y, Miyauchi A, Kihara M, Higashiyama T, Kobayashi K, Miya A. Patient age is significantly related to the progression of papillary microcarcinoma of the thyroid under observation. Thyroid. 2014;24 (1):27-34. doi:10.1089/thy.2013.0367

9. Harach HR, Franssila KO, Wasenius VM. Occult papillary carcinoma of the thyroid. A "normal" finding in Finland. A systematic autopsy study. Cancer. 1985;56(3):531-538.

10. Thorvaldsson SE, Tulinius H, Bjornsson J, Bjarnason O. Latent thyroid carcinoma in Iceland at autopsy. Pathol Res Pract. 1992;188(6):747-750. doi:10.1016/S0344-0338(11)80172-X

11. Amin MB, Edge, SB, Greene FL, et al. AJCC Cancer Staging Manual. 8th ed. New York, NY: Springer; 2016.

12. Cady B, Rossi R. An expanded view of risk-group definition in differentiated thyroid carcinoma. Surgery. 1988;104(6):947-953.

13. Hay ID, Bergstralh EJ, Goellner JR, Ebersold JR, Grant CS. Predicting outcome in papillary thyroid carcinoma: development of a reliable prognostic scoring system in a cohort of 1779 patients surgically treated at one institution during 1940 through 1989. Surgery. 1993;114(6):1050-1057, discussion 1057-1058.

14. Ito Y, Miyauchi A, Kihara M, Takamura Y, Kobayashi K, Miya A. Relationship between prognosis of papillary thyroid carcinoma patient and age: a retrospective single-institution study. Endocr J. 2012;59(5):399-405.

15. Sugino K, Kure Y, Iwasaki H, et al. Metastases to the regional lymph nodes, lymph node recurrence, and distant metastases in nonadvanced papillary thyroid carcinoma. Surg Today. 1995;25(4):324-328.

16. Anderson KL Jr., Youngwirth LM, Scheri RP, Stang MT, Roman SA, Sosa JA. T1a versus T1b differentiated thyroid cancers: do we need to make the distinction? Thyroid. 2016;26(8):1046-1052. doi:10.1089/ thy.2016.0073

17. Wang LY, Nixon IJ, Palmer FL, et al. Comparable outcomes for patients with pT1a and pT1b differentiated thyroid cancer: is there a need for change in the AJCC classification system? Surgery. 2014;156 (6):1484-1489, discussion 1489-1490. doi:10.1016/j.surg.2014.08.037

18. Tang J, Liu HB, Yu L, Meng X, Leng SX, Zhang H. Clinicalpathological characteristics prognostic factors for papillary thyroid microcarcinoma in the elderly. $J$ Cancer. 2018;9(2):256-262. doi:10.7150/jca. 22700

19. Mehanna H, Al-Maqbili T, Carter B, et al. Differences in the recurrence and mortality outcomes rates of incidental and nonincidental papillary thyroid microcarcinoma: a systematic review and meta-analysis of 21329 person-years of follow-up. $J$ Clin Endocrinol Metab. 2014;99(8):2834-2843. doi:10.1210/jc.2013-2118

20. Wei T, Li Z, Jin J, et al. Autotransplantation of Inferior Parathyroid glands during central neck dissection for papillary thyroid carcinoma: a retrospective cohort study. Int J Surg. 2014;12(12):1286-1290. doi:10.1016/j.ijsu.2014.11.001

21. Haugen BR, Alexander EK, Bible KC, et al. 2015 American Thyroid Association Management guidelines for adult patients with thyroid nodules and differentiated thyroid cancer: the American Thyroid Association Guidelines task force on thyroid nodules and differentiated thyroid cancer. Thyroid. 2016;26(1):1-133. doi:10.1089/ thy. 2015.0020
22. Morris LG, Sikora AG, Tosteson TD, Davies L. The increasing incidence of thyroid cancer: the influence of access to care. Thyroid. 2013;23(7):885-891. doi:10.1089/thy.2013.0045

23. Tam S, Boonsripitayanon M, Amit M, et al. Survival in differentiated thyroid cancer: comparing the AJCC cancer staging seventh and eighth editions. Thyroid. 2018;28(10):1301-1310. doi:10.1089/thy.2017.0572

24. Diaconescu MR, Glod M, Costea I, Clinical features surgical treatment of thyroid pathology in patients over 65 years. Chirurgia. 2016;111 (2):120-125.

25. Orimo H, Kamiya N. [Redefining the concept of elderly-for "successful aging" society]. Nippon Rinsho. 2008;66(8):1605-1614.

26. Park HS, Roman SA, Sosa JA. Treatment patterns of aging Americans with differentiated thyroid cancer. Cancer. 2010;116 (1):20-30. doi: $10.1002 / \mathrm{cncr} .24717$

27. Boltz MM, Hollenbeak CS, Schaefer E, Goldenberg D, Saunders BD. Attributable costs of differentiated thyroid cancer in the elderly Medicare population. Surgery. 2013;154(6):1363-1369, discussion 1369-1370. doi:10.1016/j.surg.2013.06.042

28. Calo PG, Medas F, Loi G, Erdas E, Pisano G, Nicolosi A. Differentiated thyroid cancer in the elderly: our experience. Int J Surg. 2014;12(Suppl 2):S140-S143. doi:10.1016/j.ijsu.2014.08.362

29. Hollenbeak CS, Boltz MM, Schaefer EW, Saunders BD, Goldenberg D. Recurrence of differentiated thyroid cancer in the elderly. Eur $J$ Endocrinol. 2013;168(4):549-556. doi:10.1530/EJE-12-0848

30. Chereau N, Tresallet C, Noullet S, et al. Prognosis of papillary thyroid carcinoma in elderly patients after thyroid resection: A retrospective cohort analysis. Medicine. 2016;95(47):e5450. doi:10.1097/MD.0000000000004864

31. Qu N, Zhang L, Ji QH, et al. Risk factors for central compartment lymph node metastasis in papillary thyroid microcarcinoma: a meta-analysis. World J Surg. 2015;39(10):2459-2470. doi:10.1007/ s00268-015-3108-3

32. Gao M, Ge M, Ji Q, et al. 2016 Chinese expert consensus and guidelines for the diagnosis and treatment of papillary thyroid microcarcinoma. Cancer Biol Med. 2017;14(3):203-211. doi:10.20892/j.issn.20953941.2017.0051

33. Zhang L, Liu Z, Liu Y, Gao W, Zheng C. The clinical prognosis of patients with $\mathrm{cN} 0$ papillary thyroid microcarcinoma by central neck dissection. World J Surg Oncol. 2015;13:138. doi:10.1186/s12957015-0553-2

34. Randolph GW, Duh QY, Heller KS, et al. The prognostic significance of nodal metastases from papillary thyroid carcinoma can be stratified based on the size and number of metastatic lymph nodes, as well as the presence of extranodal extension. Thyroid. 2012;22 (11):1144-1152. doi:10.1089/thy.2012.0043

35. Jeon MJ, Yoon JH, Han JM, et al. The prognostic value of the metastatic lymph node ratio and maximal metastatic tumor size in pathological N1a papillary thyroid carcinoma. Eur J Endocrinol. 2013;168(2):219-225. doi:10.1530/EJE-12-0744

36. Kim JY, Jung EJ, Park T, et al. Impact of tumor size on subclinical central lymph node metastasis in papillary thyroid microcarcinoma depends on age. World J Surg Oncol. 2015;13:88. doi:10.1186/ s12957-015-0478-9

37. Wang M, Wu WD, Chen GM, et al. Could tumor size be a predictor for papillary thyroid microcarcinoma: a retrospective cohort study. Asian Pac J Cancer Prev. 2015;16(18):8625-8628.

38. Moon HJ, Kim EK, Chung WY, Yoon JH, Kwak JY. Minimal extrathyroidal extension in patients with papillary thyroid microcarcinoma: is it a real prognostic factor? Ann Surg Oncol. 2011;18(7):1916-1923. doi:10.1245/s10434-011-1556-Z

39. Woo CG, Sung CO, Choi YM, et al. Clinicopathological significance of minimal extrathyroid extension in solitary papillary thyroid carcinomas. Ann Surg Oncol. 2015;22(Suppl 3):S728-S733. doi:10.1245/s10434-015-4659-0 
40. Hu G, Zhu W, Yang W, Wang H, Shen L, Zhang H. The effectiveness of radioactive iodine remnant ablation for papillary thyroid microcarcinoma: a systematic review and meta-analysis. World J Surg. 2016;40(1):100-109. doi:10.1007/s00268-015-3346-4

41. Kwon H, Jeon MJ, Kim WG, et al. Lack of efficacy of radioiodine remnant ablation for papillary thyroid microcarcinoma: verification using inverse probability of treatment weighting. Ann Surg Oncol. 2017;24(9):2596-2602. doi:10.1245/s10434-017-5910-7
42. Lee J, Park JH, Lee CR, Chung WY, Park CS. Long-term outcomes of total thyroidectomy versus thyroid lobectomy for papillary thyroid microcarcinoma: comparative analysis after propensity score matching. Thyroid. 2013;23(11):1408-1415. doi:10.1089/ thy. 2012.0463

43. Durante C, Montesano T, Torlontano M, et al. Papillary thyroid cancer: time course of recurrences during postsurgery surveillance. J Clin Endocrinol Metab. 2013;98(2):636-642. doi:10.1210/jc.2012-3401

\section{Publish your work in this journal}

Cancer Management and Research is an international, peer-reviewed open access journal focusing on cancer research and the optimal use of preventative and integrated treatment interventions to achieve improved outcomes, enhanced survival and quality of life for the cancer patient.
The manuscript management system is completely online and includes a very quick and fair peer-review system, which is all easy to use. Visit http://www.dovepress.com/testimonials.php to read real quotes from published authors. 